the formulas (11), (8), and (5) furnish us a solution of the problem.

We still have to consider the possibility of satisfying the equations (4) by making vanish more than one of the expressions in brackets in the left hand sides of these equations and less than two of the numbers $a, b, c, d$; but it is easy to see that this is possible only in the exceptional case mentioned above, namely, where two of the numbers $f, g, h, k$ are equal. Barring this exceptional case we have then a unique system of values for $e, a^{2}, b^{2}, c^{2}$, and $d^{2}$; but we can choose the signs of $c$ and $d$.

The University of Michigan

\title{
ON THE PHRAGMÉN-BROUWER THEOREM*
}

\author{
BY W. A. WILSON
}

1. Introduction. The purpose of this note is to give an elementary demonstration of the validity of the PhragménBrouwer theorem in spaces satisfying certain conditions. This theorem has been proved and generalized by Urysohn and Alexandroff $\dagger$ for cartesian spaces by means of the theory of dimensionality, but the great importance of the theorem seems to the writer to justify the offering of another proof which involves only the elementary principles of the point-aggregate theory. As is well known, to prove this theorem for a space is the same as demonstrating that, if $m$ and $n$ are any two points of the space and $C$ is an irreducible cut between $m$ and $n$, then $C$ is a continuum. It is this form which will be used and the proof is deduced from the validity of the theorem in the euclidean plane.

* Presented to the Society, October 26, 1929. Shortly after the submission of the manuscript of this note to the editors, C. Kuratowski published another proof of this theorem in the Fundamenta Mathematicae, vol. 14, pp. 304-310. This paper was not withdrawn, because the great importance of the theorem seemed to the author to warrant the belief that another demonstration would not be void of interest to readers.

$\dagger \mathrm{P}$. Alexandroff, Sur les multiplicités cantoriennes et le théorème de PhragménBrouwer généralisé, Comptes Rendus, vol. 183, pp. 722-724. 
2. Notation. In addition to the familiar notations of the pointaggregate theory, the following will be used.

If $a$ and $b$ are two points, the distance between them is denoted by Dist $(a, b)$.

The symbol $V_{\epsilon}(a)$ denotes the set of points whose distances from $a$ are less than $\epsilon$.

If $C$ is a closed cut of space between the points (or point sets) $A$ and $B$, we write that $C$ is an $S(A, B)$.

The symbol $\overline{\lim } A_{i}$ denotes the upper closed limiting set of the sequence of sets $\left\{A_{i}\right\}$ as $i \rightarrow \infty$.

3. Convex Spaces. Let $a$ and $b$ be points of a metric space $Z$ and let $k=$ Dist $(a, b)$. For each value of $t, 0 \leqq t \leqq k$, let there be one and only one point $x$ such that Dist $(a, x)=t$ and Dist $(x, b)=$ $k-t$. If this property pertains to each pair of points of $Z$, we say that $Z$ is convex. ${ }^{*}$ The set of points $\{x\}$ is called a linear segment, or simply a segment, and is denoted by $a b$. Thus a convex space is one in which each pair of points determines a unique segment.

Every euclidean space is convex, but not conversely. As an example, consider a space consisting of three ordinary linear segments $a x, b x$, and $c x$ lying in a euclidean plane. Let the distance between a pair of points on any one of these be measured as usual. If $u, v, w$ are points on $a x, b x$, and $c x$, respectively, let Dist $(u, v)$ be defined as Dist $(u, x)+\operatorname{Dist}(v, x)$, etc. In this space any two points can be joined by a unique segment, but we notice that two different segments, as $u v$ and $u w$, may have a segment, here $u x$, in common.

The following properties are readily proved for any convex space in which every bounded set has at least one limiting point:

(a) it is a continuum;

(b) it is locally connected at each point;

(c) if $\left\{x_{i}\right\}$ and $\left\{y_{i}\right\}$ are sequences of points converging to $x$ and $y$, respectively, Dist $\left(x_{i}, y_{i}\right) \rightarrow \operatorname{Dist}(x, y)$ and $x_{i} y_{i} \rightarrow x y$, that is, the set $x y$ is both the upper and the lower closed limiting set of the sequence of sets $\left\{x_{i} y_{i}\right\}$.

A space of this kind obviously includes euclidean space of any number of dimensions as a special case.

* This term is used in a slightly more restricted sense by Hausdorff; see Mengenlehre, p. 96. 
4. Theorem. Let $Z$ be a convex space in which every bounded infinite set has at least one limiting point. If $m$ and $n$ are two points and $C$ is an irreducible $S(m, n)$, then $C$ is a continuum.

Proof. We assume the opposite and arrive at a contradiction. Let $C=A+B$, where $A$ and $B$ are closed and $A \cdot B=0$. Let $M$ and $N$ be the components of $Z-C$ containing $m$ and $n$, respectively. Since $Z$ is everywhere locally connected, $M$ and $N$ are connected regions and $C$ is the frontier of each of them.

Let $h$ be a point of $A$ and $k$ one of $B$, and let $\epsilon>0$ be less than one third of the smaller of the quantities Dist $(h, B)$ and Dist $(k, A)$. Then $B \cdot V_{\epsilon}(h)=0=A \cdot V_{\epsilon}(k)$. Now there is a point $a$ in $M$ and a point $c$ in $N$ such that the segment $a c \subset V_{\epsilon}(h)$. Likewise there is a point $b$ in $M$ and a point $d$ in $N$ such that the segment $b d \subset V_{\epsilon}(k)$. Then $a c \cdot B, b d \cdot A$, and $a c \cdot b d$ are void. As $C$ is an $S(m, n)$, we have $a c \cdot A \neq 0$ and $b d \cdot B \neq 0$. Since $Z$ is everywhere locally connected, there are simple arcs $a b$ and $c d$ in $M$ and $N$, respectively. Finally, there is no loss in generality in assuming that $(a b+c d) \cdot(a c+d b)=a+b+c+d$. For $a c \cdot a b$ and $a c \cdot c d$ are closed sets; hence $a c$ contains a segment $a^{\prime} c^{\prime}$ such that $a^{\prime} c^{\prime} \cdot a b=a^{\prime}$ and $a^{\prime} c^{\prime} \cdot c d=c^{\prime}$. Likewise, $b d$ contains a segment $b^{\prime} d^{\prime}$ such that $b^{\prime} d^{\prime} \cdot a b=b^{\prime}$ and $b^{\prime} d^{\prime} \cdot c d=d^{\prime}$. Let $a^{\prime} b^{\prime}$ be the sub-arc of $a b$ whose ends are $a^{\prime}$ and $b^{\prime}$, and $c^{\prime} d^{\prime}$ have a similar relation to $c d$. Clearly $a^{\prime} b^{\prime}, a^{\prime} c^{\prime}, b^{\prime} d^{\prime}$, and $c^{\prime} d^{\prime}$ have the properties assigned to $a b, a c, b d$, and $c d$.

In a cartesian plane let $S$ be the closed square whose frontier is the union of the segments $p q, p r, q s$, and $r s$, each of unit length. Set up a homeomorphism between $a b$ and $p q$, and between $c d$ and $r s$, so that $a, b, c$, and $d$ correspond to $p, q, r$, and $s$, respectively. Now let $u$ be any point of $p q$ and $v$ one of $r s$ so that $u v$ is parallel to $p r$. In $Z$ let $x$ and $y$ be the points of $a b$ and $c d$ corresponding to $u$ and $v$, respectively, and let $x y$ be the segment joining them. (If $u=p, x y=a c$; if $u=q, x y=b d$.) Let $Q$ be the union of the sets $\{x y\}$ for all positions of $u$ on $p q$. Set up a homeomorphism between each $x y$ and the corresponding $u v$ such that, if $z$ is a point of $x y$ and $t$ is the corresponding point of $u v$, Dist $(u, t)=\mathrm{Dist}(x, z) / \mathrm{Dist}(x, y)$. Then to each point of $S$ there corresponds exactly one point of $Q$. In general the converse is not true, for two or more different segments $\{x y\}$ may have common points. 
We proceed to prove that $Q$ is the continuous image of $S$. Let $t_{i} \rightarrow t$ in $S$ and let $z_{i}$ and $z$ be the corresponding points in $Q$; we must prove that $z_{i} \rightarrow z$. Let $t_{i}$ lie on $u_{i} v_{i}, t$ lie on $u v, x_{i} y_{i}$ correspond to $u_{i} v_{i}$, and $x y$ correspond to $u v$. It is obvious that $u_{i} \rightarrow u, v_{i} \rightarrow v$, whence $x_{i} \rightarrow x, y_{i} \rightarrow y$, and $x_{i} y_{i} \rightarrow x y$. Hence, whether or not the sequence $\left\{z_{i}\right\}$ converges, $\overline{\lim } z_{i} \subset x y$.

By construction Dist $\left(x_{i}, z_{i}\right)=\operatorname{Dist}\left(x_{i}, y_{i}\right) \times$ Dist $\left(u_{i}, t_{i}\right)$. By the previous paragraph Dist $\left(x_{i}, y_{i}\right) \rightarrow$ Dist $(x, y)$ and also Dist $\left(u_{i}, t_{i}\right) \rightarrow$ Dist $(u, t)$. Since $Q$ is bounded, the sequence $\left\{z_{i}\right\}$ has a convergent sub-sequence $\left\{z_{i}{ }^{\prime}\right\}$ whose limit we call $\boldsymbol{z}^{\prime}$. The results just obtained show that Dist $\left(x, z^{\prime}\right)=$ Dist $(x, y)$ $\times$ Dist $(u, t)$, or Dist $(u, t)=\operatorname{Dist}\left(x, z^{\prime}\right) /$ Dist $(x, y)$. The definition of the correspondence between $Q$ and $S$ gives immediately that $z^{\prime}=z$. As this holds for every convergent sub-sequence of $\left\{z_{i}\right\}$, it follows that $z_{i} \rightarrow z$ and hence that the correspondence is continuous.

Let $A^{\prime}=A \cdot Q$ and $B^{\prime}=B \cdot Q$. Then $A^{\prime} \cdot b d=0, B^{\prime} \cdot a c=0$, and $\left(A^{\prime}+B^{\prime}\right) \cdot(a b+c d)=0$. Let $E$ be the set of all the points of $S$ whose images in $Q$ are points of $A^{\prime}$, and let $F$ have a like relation to $B^{\prime}$. Then $E \cdot p r \neq 0, F \cdot q s \neq 0, E \cdot q s=0, F \cdot p r=0$ and $(E+F) \cdot(p q+r s)=0$. Since the correspondence is continuous, $E$ and $F$ are closed; since $A^{\prime} \cdot B^{\prime}=0, E \cdot F=0$.

As $E+F$ does not contain a continuum joining $p r$ to $q s$, it follows from the properties of the euclidean plane that there is a continuum $K$ in $S-(E+F)$ such that $K \cdot r s \neq 0$ and $K \cdot p q \neq 0$. Let $L$ be the image of $K$ in $Q$. Then $L \cdot\left(A^{\prime}+B^{\prime}\right)=0$ and $L$ is a continuum. As $L \subset Q, L \cdot(A+B)=0$. Since $K \cdot r s \neq 0$ and since $K \cdot p q \neq 0, L \cdot a b \neq 0$ and $L \cdot c d \neq 0$.

But $a b \subset M$ and $c d \subset N$, whence it follows that $C=A+B$ is not an $S(M, N)$, contrary to hypothesis. Thus the theorem is proved.

YALE UNIVERSITY 